\title{
Baumkronenwelten Teil 1: \\ Epiphytische Farne im Alten Botanischen Garten der Universität Göttingen
}

\author{
Michael SCHWerdTfeger
}

\begin{abstract}
In the Old Botanical Garden of the University of Göttingen tropical (epiphytic) ferns are traditionally cultivated. Since the restoration over 10 years ago the collection of ferns was enlarged and new displayed. Some species and their cultivation are explained.
\end{abstract}

\section{Zusammenfassung}

Im alten Botanischen Garten der Universität Göttingen werden traditionell tropische (epiphytische) Farne kultiviert. Seit der Renovierung des historischen Farnhauses vor über 10 Jahren wurde die Farnsammlung erweitert und neu präsentiert. Einige markante Arten und ihre Kulturbedingungen werden vorgestellt.

\section{Exotische Pflanzen im Universitätsgarten des Albrecht von Haller}

„... das Leben findet einen Weg" sagt der Chaosforscher Ian Malcolm in STeven SpielBERGs Jurassic Park und sieht damit die Dynamik von Lebwesen voraus, die außer Kontrolle geraten. Im Falle von Jurassic Park sind es aus fossiler DNA geschaffene Dinosaurier, die die menschliche Obhut und Kontrolle verlassen. Im vorliegenden Artikel geht es um tropischepiphytische Farne in unseren Gewächshäusern, die zwar weniger spektakulär als Dinosaurier sind, aber Zeitgenossen der Dinosaurier waren und noch heute ganz real sind.

Der Botanische Garten der Universität Göttingen wurde 1737 von Albrecht von HaLLER gegründet, der dank internationaler Kontakte die Sammlungen des Gartens mehrte und bereits 1743 , wenige Jahre nach der Gründung des Gartens, eine „Brevis enumeratio stirpium horti Goettingensis" mit ca. 1500 Pflanzenarten vorlegte. $\mathrm{Zu}$ jener frühen Zeit war die Welt noch groß und aufregend, das Reisen gefährlich und die Göttinger „Palma hortensis“" (die europäische Zwergpalme Chamaerops humilis, die in Spanien und Italien wild wächst) eine viel bewunderte Rarität. Über seine holländischen Gewährsleute hatte Haller auch bereits einige kostbare Kap-Pflanzen erhalten, darunter die

Abb. 1: Campyloneurum angustifolium.

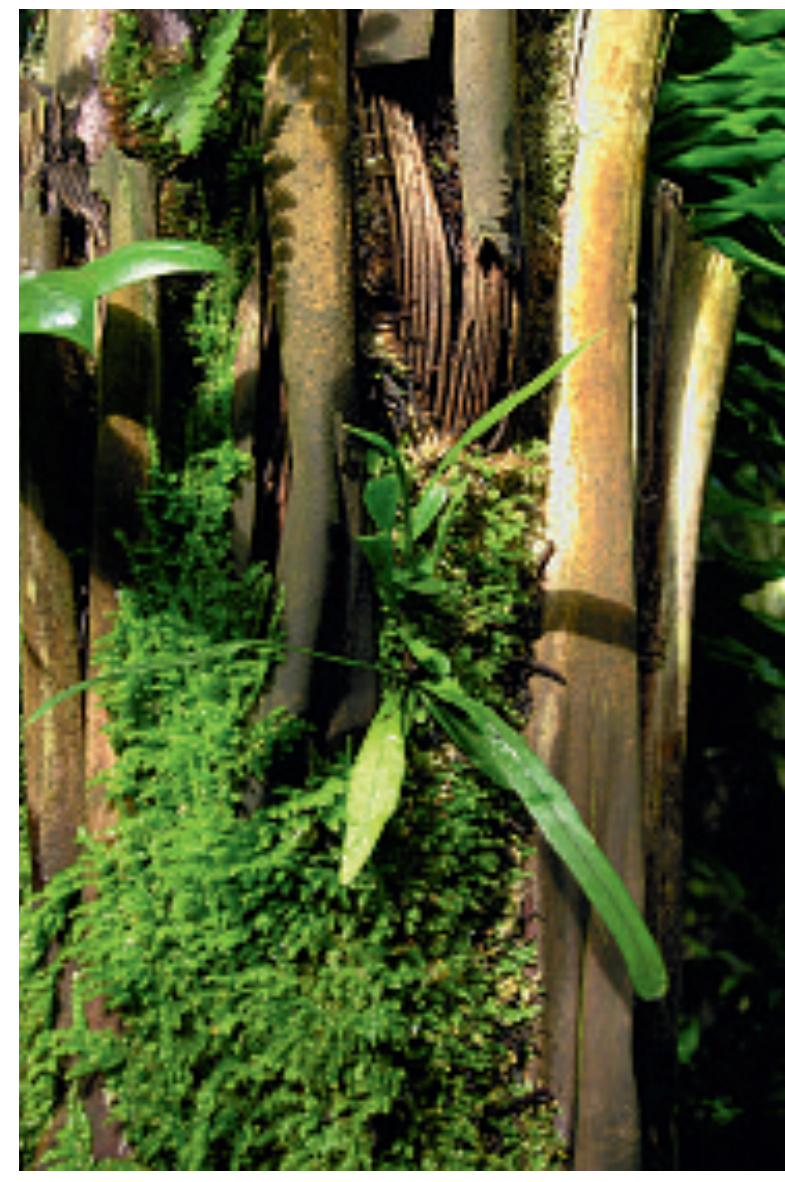

ersten Pelargonien, Aloe und Gasteria. Ein Stich aus dem Jahre 1747 zeigt einen ersten, an den Stadtwall geschmiegten "Gewächs-Saal“ für die Unterbringung von HaLlers Exoten.

Um 1830 wurden unter der Gärtnerdynastie Fischer, die Verbindungen zu den WendLANDS in Herrenhausen pflegten, zwei herrli- 

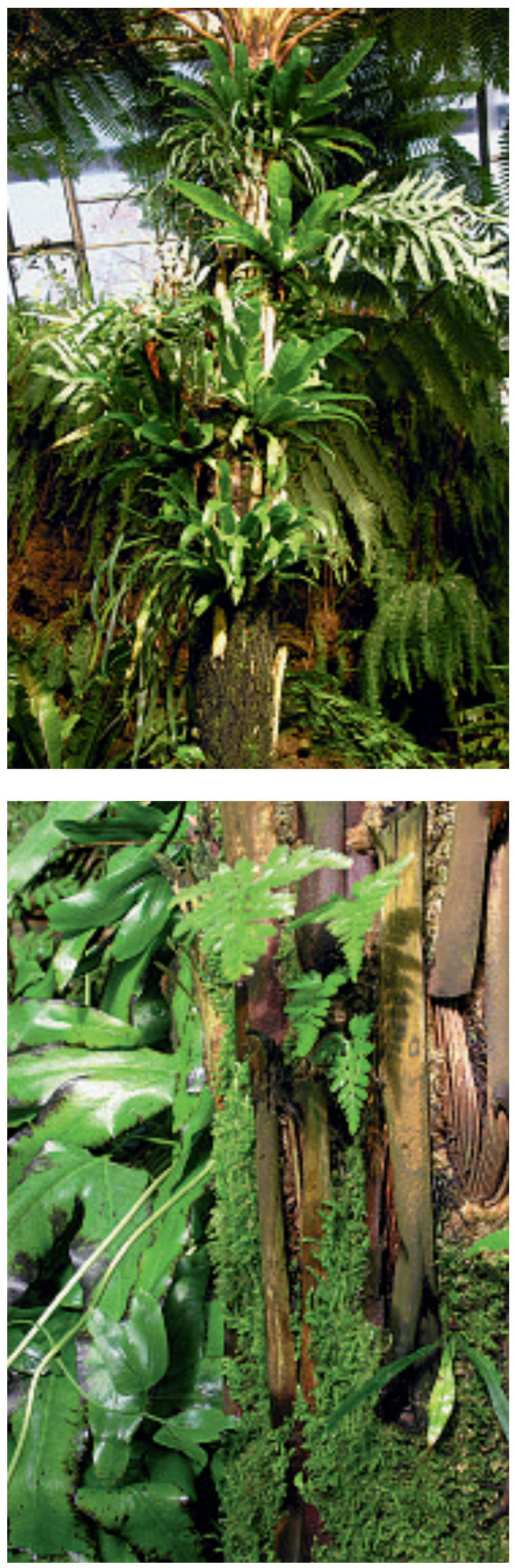

che Gewächshäuser gebaut und mit tropischen Pflanzen bestückt (Diese Gewächshäuser stehen heute noch!). Im Jahre 1857 wurde eine Orangerie im viktorianischen Stil für Kübelpflanzen errichtet. Diese wurde 1910 unter dem legendären Gartenmeister CARL BonstedT mit Lavatuff, Grotte und Wasserbecken zum Farnhaus umgestaltet. Nachdem das Farnhaus 1999-2000 mit Hilfe der Göttinger Öffentlichkeit und Mitteln des Landes Niedersachsen restauriert werden konnte, verfügt der Garten auch heute über drei historische Gewächshäuser. Das so genannte Erikenhaus beherbergt aktuell überwiegend Kanarenpflanzen. Das Araceenhaus ist den Anthurien, Philodendren, Myrmecodien, Dischidien, Orchideen und anderen Epiphyten tropischer Tieflandregenwälder gewidmet. Seit genau hundert Jahren dient das Farnhaus der Präsentation tropischer Farnpflanzen aus aller Welt. Es ist eine Herausforderung, hier jenseits des üblichen Sortiments ganz besondere tropische Farne zu kultivieren sowie sich mit ihrer Vermehrung zu beschäftigen.

\section{Wiedereinzug tropischer Farne in das restaurierte Farnhaus}

Im Jahre 2000 war die Restaurierung des Farnhauses abgeschlossen. Nach Beendigung der Bauarbeiten bestand die Chance, das historische Gewächshaus mit einem ansprechenden Sortiment tropischer Farne völlig neu zu bepflanzen. Die jahrzehntealten „Göttinger Originale", darunter drei mächtige Angiopteris-Exemplare und eine Aglaomorpha heraclea, hatten die Bauarbeiten im sicheren Exil verbracht und kehrten nun zurück. Gesellschaft bekamen sie durch zahlreiche Neulinge: Auf Anregung der Gärtnerschaft war die Anzucht von Farnen aus Sporen in Angriff genommen worden und hatte mittlerweile beachtliche Erfolge erbracht (Schwerdtfeger 2009). Knapp 100 Arten konnten nun das Farnhaus wieder besiedeln.

Abb. 2 (oben): Blick in das Farnhaus.

Abb. 3 (unten): Viele Farne säen sich im Farnhaus von selbst aus. 
Die noch von BornstedT errichteten „Felswände" aus Eifel-Lava erwiesen sich bei ausreichender Feuchtigkeit als ideales Farnsubstrat. In dem nach den Bauarbeiten völlig kahlen Farnhaus wurden Initialpflanzungen getätigt; den Rest besorgten die Farne durch Sporenaussaat von selbst. Vor unseren Augen lief die Besiedelung von vegetationslosem, sterilen Boden durch Farnpflanzen ab. Dass die Farne bald beginnen würden, sich auf der feucht-porösen Lavaoberfläche durch Sporenaussaat selbst zu verbreiten, war von vornherein einkalkuliert und erwünscht. Es wurde darauf verzichtet, von großen, formenreichen Gruppen wie Adiantum oder Nephrolepis zahlreiche, schwer unterscheidbare Arten und Sorten zu verwenden, die sich bald unkontrollierbar vermehren und hybridisieren würden.

\section{Ein besonderer Schwerpunkt: Epiphytische Farne}

Schon vor hundert Jahren war der „Königlich Botanische Garten“ in Göttingen für seine exquisiten Warmhauskulturen bekannt. 1901 trat der damals noch sehr junge CARL Bonstedt in die Dienste des Botanischen Gartens. Zwei Jahre später wurde ein Viktoriahaus gebaut (der heute an gleicher Stelle stehende Nachfolger ist leider kleiner und weniger prachtvoll). Bonstedt machte sich als Züchter von Fuchsien, Sarracenien, Nepenthes und anderen Pflanzengruppen und vor allem als Herausgeber des Standardwerkes „Pareys Blumengärtnerei“ einen Namen. Noch heute ist der Garten im Besitz hunderter gestochen scharfer Glas-Fotoplatten, die Porträts seiner erlesenen Pflanzen zeigen und die von den überragenden gärtnerischen Fähigkeiten des Gartenmeisters zeugen.

In diesem Zusammenhang interessieren besonders die Porträts tropisch-epiphytischer Farne wie Microgramma, Drynaria und Aglaomorpha, die damals allesamt noch in der riesigen Gattung Polypodium vereinigt waren. Bonstedt war von der Kultur der Epiphyten in Töpfen abgekommen. Seine Fotos zeigen herrlich entwickelte Epiphyten in Hängekörben, auf moosumwickelten Hölzern und auf Baumfarnbrettchen.
Auf den historischen Aufnahmen fällt der hervorragende Kulturzustand auf. Über die von BonstedT erreichten Kulturbedingungen geben besonders seine berühmten Nepenthes-Exemplare Auskunft. Diese Pflanzen bilden bekanntlich viele und schöne Kannen nur dann aus, wenn die Luftfeuchtigkeit niemals signifikant absinkt. Dies erfordert im Sommer bei offener Lüftung und auch im Winter während der Heizperiode eine besonders sorgfältige Klimatisierung. Die herrlichen Kannenpflanzen von Carl Bonstedt geben Zeugnis davon, dass das Aufrechterhalten hoher Luftfeuchte ihm meisterhaft gelang. In Zeiten des Ersten Weltkrieges, ohne elektronisch gesteuerte Sprühnebelanlage, müssen wir uns einen aus heutiger Sicht hohen Personaleinsatz vorstellen: Mehrmals täglich musste per Hand gesprüht und genebelt oder ein "Aufguss" vorbereitet werden.

Auch wenn in den 80 Jahren nach Gartenmeister Bonstedt viele Spuren verblassten, Kulturen verschwanden und wieder aufgebaut wurden, so lassen sich noch heute Spuren aus dieser Phase finden und Verbindungen zu den Pflanzenkulturen der Göttinger Jugendstilzeit herstellen. Die noch vorhandenen Farne wurden im Zuge der Neubestückung des restaurierten Farnhauses bearbeitet: Namenlose wurden bestimmt, unschön gewordene verjüngt bzw. vermehrt. Zusätzlich wurden in großem Umfang aus anderen Sammlungen im In- und Ausland Sporen besorgt und somit die Geschichte der epiphytischen Farne in Göttingen um ein neues Kapitel erweitert. Die ergiebigste Quelle für exotische Farne stellte die hervorragende Farnsammlung des Botanischen Gartens München dar. Teilstücke spektakulärer epiphytischer Farne wie Platycerium, Drynaria und Aglaomorpha konnten in Göttingen Einzug halten. Und bald darauf trug der Wiederaufbau der Farnsammlung auch respektable wissenschaftliche Früchte: Der Pteridologe Harald Schneider publizierte - teilweise auf Grundlage der Göttinger Farnsammlung - in hochrangigen Journalen und sogar in Nature (Schneider et al. 2004). 


\section{Epiphytische Farne gedeihen und verwildern im Botanischen Garten}

Hundert Jahre nach BonstedT gedeihen nach wie vor - und mehr denn je - epiphytische Farne im Botanischen Garten Göttingen. Große, robuste Arten wurden im Farnhaus ausgepflanzt, zum Bekleiden der Lavawände verwendet, auf die neuen Epiphytenstämme gesetzt oder in Ampeln aufgehängt. Überschüssige Pflanzen wurden, sofern geographisch passend, auch auf den Epiphytenstämmen im Regenwaldhaus angesiedelt.

Die Epiphytenstämme in beiden Häusern sind aus Beton, was weder dem Wohlbefinden der Pflanzen noch der Optik Abbruch tut: Bei entsprechender Klimatisierung bedeckt sich das profane Material bald mit Algen und Moos, und die Epiphytengesellschaften wachsen wie in der Natur durcheinander und kaschieren bald vollständig das unschön-leblose Material. Besonders praktisch lassen sich die üppig bewachsenen Betonstämme bei Führungen einsetzen: Werden doch die auf den Ästen wach- senden Orchideen, Bromelien, Peperomien und Farne von Laien oft als Schmarotzer oder Parasiten angesehen. Der Charakter der Epiphyten lässt sich mit nichts besser demonstrieren als mit ihrem Wachstum auf leblosem Beton.

Feinere, kostbarere epiphytische Farne, die im Farnhaus untergehen würden, fanden ihren Platz im Araceenhaus, wo jetzt Davallia, Pyrrosia, Microgramma und Lecanopteris, auf Holzknüppeln aufgebunden, zusammen mit Anthurium, Nepenthes und Myrmecodia gedeihen. Das kleine, nicht öffentlich zugängliche Orchideenhaus dient als Kinderstube für besonders kostbare kleine Platycerien, tropisch-epiphytische Bärlappe, Belvisia und Solanopteris.

Bonstedt hatte in Ermangelung moderner Technik seine Erfolge offenbar durch ehrliche Handarbeit in Form von fleißigem Nebeln und Sprühen erreicht. Der Schlüssel zum Erfolg bei der Epiphytenkultur ist bekanntlich ein kontinuierlich mild-feuchtes, aber keinesfalls dauernasses Substrat, regelmäßige Befeuchtung der Oberflächen und eine konstant hohe Luft-

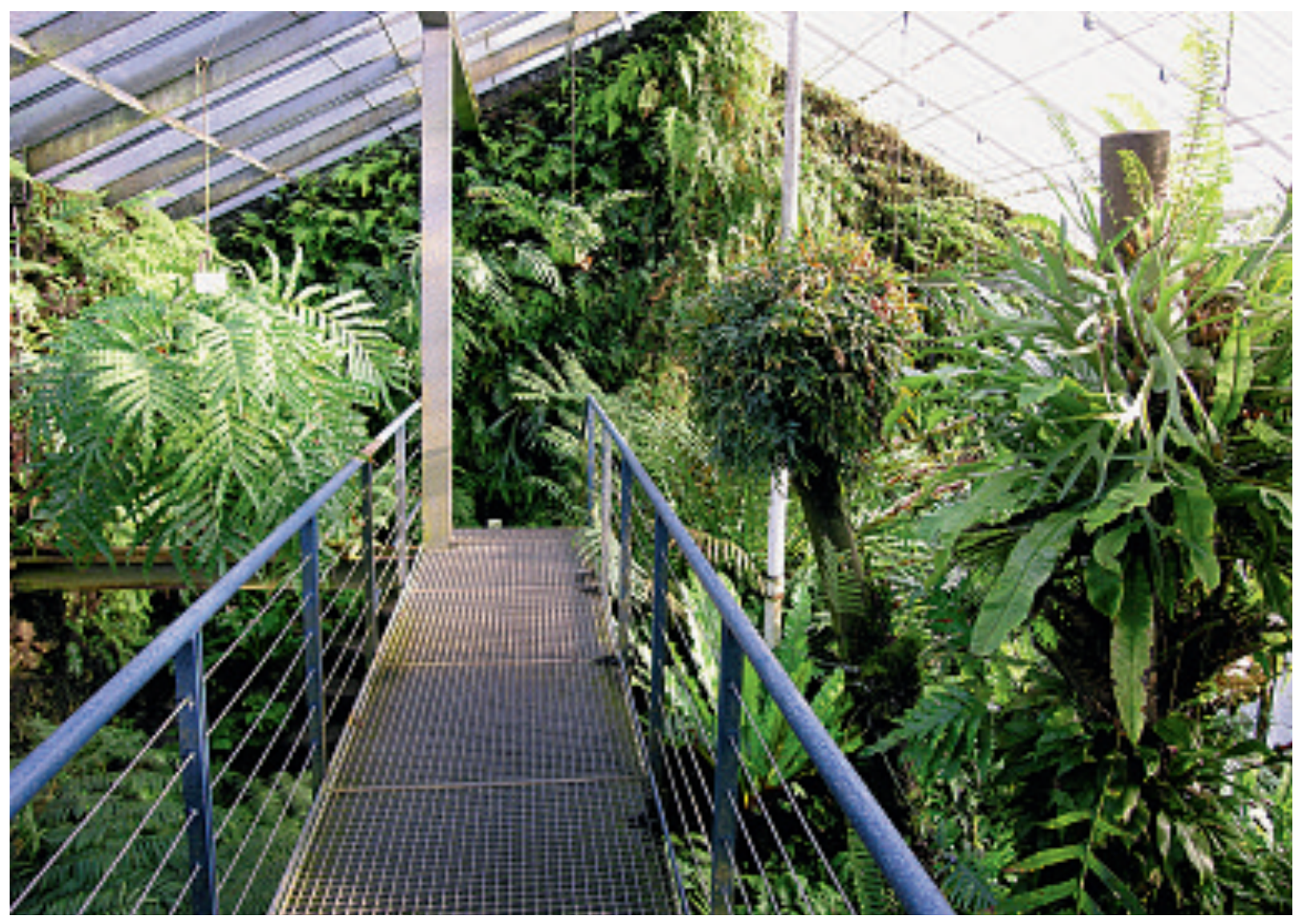


feuchtigkeit. Mit anderen Worten bedeutet dies: Möglichst hohe Feuchtigkeit bei möglichst wenig Nässe.

Auch heute ist die Klima-Technik in den Göttinger Gewächshäusern eher einfach. Was hier jedoch an Technik fehlt, wird durch große Professionalität, Engagement und Einsatz der Gärtnerinnen und Gärtner wettgemacht. Dass sie auch heute ein ideales Epiphytenklima zu schaffen vermögen, wird besonders dadurch deutlich, dass mehr und mehr epiphytische Farne zu verwildern beginnen. Aus Sporen spontan gekeimte Jungpflanzen erscheinen an den Lavawänden, den faserigen Stämmen der Baumfarne, als Untermieter in den großen Horsten der Geweihfarne oder gar in unerreichbarer, luftiger Höhe an den dünnen Seilen, an denen die großen Epiphyten-Solitäre aufgehängt sind. Und wie viel eindrucksvoller wirkt doch ein Epiphyt, wenn er nicht gepflanzt aussieht, sondern sich offenbar selber an einem von der Decke gespannten Seil angesiedelt hat!

Folgende epiphytische Farne sind im Farnhaus bisher durch spontane Sporenkeimung verwildert: Asplenium nidus, Asplenium australasicum, Microsorum musifolium, Niphidium crassifolium, Campyloneurum angustifolium, Nephrolepis pendula und Phlebodium aureum. Mit der Gärtnerschaft wurde vereinbart, im Farnhaus der Entwicklung einer quasi-natürlichen Vegetation in Grenzen freien Lauf zu lassen. Spontan angesiedelte Farne werden also nur dort entfernt, wo sie Kostbareres bedrängen und gefährden.

Das Araceenhaus ist kleiner und niedriger als das Farnhaus und beherbergt neben Anthurien und Philodendron auch Maranten, Melastomataceen und andere Pflanzen tropischer Tieflandregenwälder. Besonders sorgfältig wird hier auf ein Aufrechterhalten „regenwaldartiger“ Luftfeuchtigkeit geachtet, da vor allem die hier untergebrachten Nepenthes ein Absinken der Luftfeuchtigkeit unverzüglich mit vertrockne-

Abb. 4 (Seite 98): Blick über Epiphytenstämme mit prächtigen Farnen.

Abb. 5 (oben): Huperzia squarrosa.

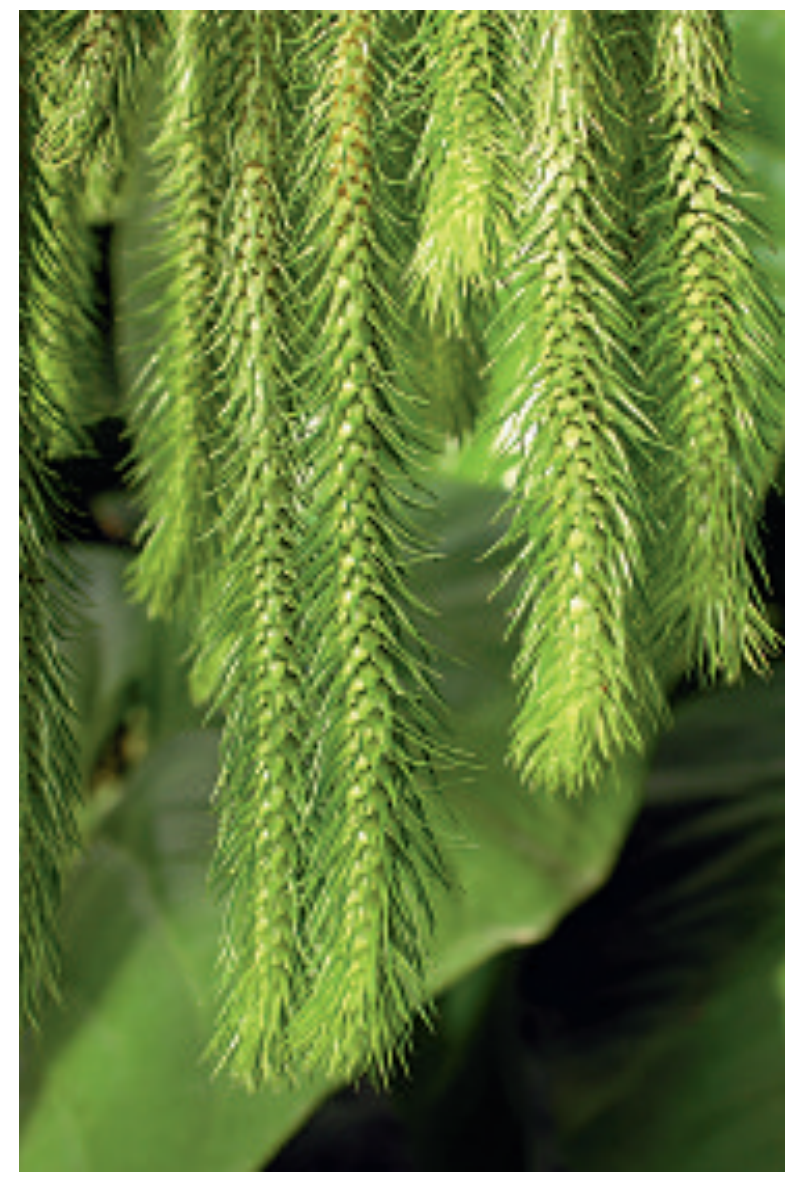

ten Kannenblättern quittieren. In diesem besonders luftfeuchten Klima verwildern bisher Microsorum musifolium, Pecluma eurybasis, Polypodium sanctae-rosae, Vittaria lineata, Microsorum scolopendrium, Platycerium bifurcatum und Campyloneurum angustifolium. Die letztere, sehr elegante Art beginnt hier einen invasiven Charakter an den Tag zu legen: In den Orchideenkörbchen und in den kostbaren Lecanopteris-Farnen bildet sie schnell große Horste und muss entfernt werden. Besonders gut gedeiht sie auf den so genannten „Gießtöpfen" (unglasierte, wassergefüllte Tontöpfchen), die hier mit großem Erfolg für besonders feuchtigkeitsliebende Epiphyten verwendet werden. Bei einer unlängst durchgeführten Bestandsaufnahme wurden einige spektakuläre Campyloneurum-Schaustücke entdeckt, die sich als spontane Invasoren solcher Gießtöpfe entpuppten, auf denen sie die eigentlichen, zarten Bewohner längst spurlos erdrückt hatten.

Im Orchideenhaus hat sich ein besonders erstaunlicher Farn (Belvisia spicata) selbständig 

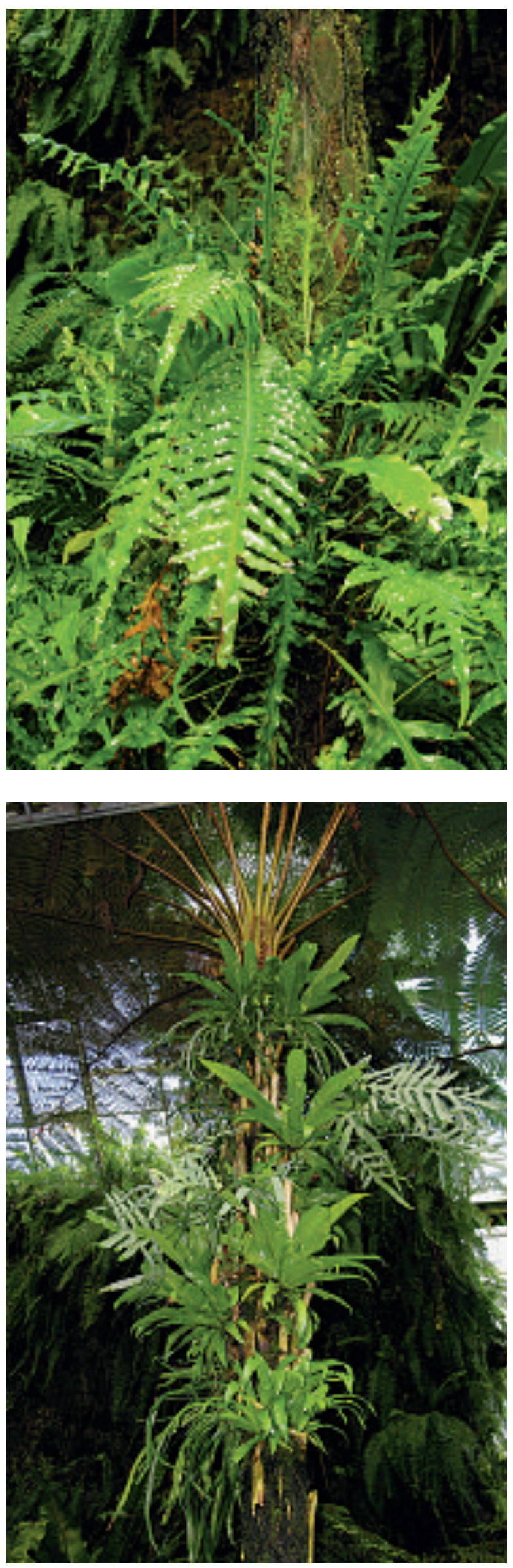

gemacht. Vor 10 Jahren angezogen und in kleine Ampeltöpfchen gepflanzt, bildeten die Pflanzen ihre lanzettlichen, bandförmigen Blätter und bald auch ihre schmalen, sporentragenden Bereiche, die wie ein dünnes Mäuseschwänzchen dem sterilen Blattteil aufsitzen. Dieser kleine, unverwechselbare Farn erscheint neuerdings in den Horsten der am Block kultivierten Orchideen und wird geduldet, so lange er seine Gastgeber nicht gefährdet.

\section{Erstaunlich widerstandsfähig: der „Unkrautfarn“ Pblebodium aureum}

Einer der epiphytischen Farne hat sich in mehreren Häusern als potentielle Problempflanze entwickelt: Phlebodium aureum (= Polypodium aureum) ist mit seinen grob fiederschnittig gelappten, matt blaugrauen Blättern zweifellos sehr attraktiv und daher auch im Zierpflanzensortiment unserer Gartencenter vertreten. Das auf der Substratoberfläche kriechende Rhizom ist gut daumenstark, spricht uns durch seine weich-zottige, goldbraune Behaarung an und hat dem Farn bei uns den Spitznamen „Katzenpfötchen“ gegeben. Die Rhizome kriechen auf den Epiphytenstämmen entlang und entwickeln dekorative Blattspreiten von $1 \mathrm{~m}$ Länge auf ebenso langem Stiel. Wenn der Farn diese Größe erreicht hat, ist er fertil. Die regelmäßig angeordneten Sori (= Sporangienhäufchen) entlassen Unmengen Sporen von ungeheurer Vitalität. Der Farn keimt an allen luftig-epiphytischen Standorten. Diese sind beispielsweise das Rindensubstrat der Anthurien und Orchideen, die Ampeltöpfe der Gesnerien, Hoyas und Myrmecodien oder die Blöcke mit aufgebundenen kleinen Epiphyten-Kostbarkeiten. Besonders auffällig ist dabei die große Resistenz des Farns gegenüber Austrocknung. Viele PolypodiumVerwandte sind an wechselfeuchte Bedingungen angepasst, und vom tropisch-amerikanischen Polypodium polypodioides („Resurrection

Abb. 6 (oben): Microsorum punctulatum.

Abb. 7 (unten): Asplenium antiquum und Phlebodium areolatum.

Abb. 8 (Seite 101): Phlebodium aureum. 
fern") ist bekannt, dass die Blätter auch nach $97 \%$ Wasserverlust und völligem Einrollen nach Befeuchtung wieder auferstehen. Phlebodium aureum scheint ebenfalls und schon als Sämling sehr austrocknungsresistent zu sein. Dieser Farn keimt in unseren Gewächshäusern selbst in den „Baumkronen“ der Epiphytenstämme, die ganztags volles Licht erhalten und vom Gießen und Sprühen nur mit Mühe erreicht werden. Zeugnis für seine Vitalität lieferte ein unfreiwilliges Trockenexperiment: Bei der Neugestaltung des Regenwaldhauses wurden über dem Weg große Epiphyten-Arrangements aus Cattleyen, Bromelien, Gesneriaceen und Farnen aufgehängt, die sich gut entwickelten und dem Besucher ein eindrucksvolles Bild von den „hängenden Gärten" der Epiphyten vermitteln. Einer dieser bepflanzten Äste wurde so hoch im freien, vollsonnigen Luftraum aufgehängt, dass sich ein ausreichendes Feuchthalten als schwierig erwies und selbst eine große, xeromorphe Aechmea mittlerweile verdorrt ist. Dort gedeiht aber ganz vital ein selbst angesiedeltes Phlebodium aureum, das sich dort in voller Sonne und bei kärglicher Bewässerung zu einem Schaustück von 1,5 m Spannweite entwickelt hat. Von seinem schwer erreichbaren Sitz aus bestreut dieser Farn das ganze Regenwaldhaus mit unerwünschten Sporen.

Im Araceenhaus mit seinem besonders luftfeuchten Klima waren vor Jahren etliche starkwüchsige Orchideen (Stanhopea, Vanda, Angraecum) in große Lattenkörbe gepflanzt worden. In der klassischen Orchideenkultur soll das Substrat luftig, aber wasserspeichernd sein und so die Orchideenwurzeln vor Austrocknung schützen. Seither haben das Gärtnerteam und die Kulturmethoden gewechselt, ein Aufrechterhalten der nötigen Luftfeuchte gelingt noch besser als zuvor, und mittlerweile gedeihen viele Orchideen in Blockkultur und zeigen durch üppigen Besatz mit weißen Luftwurzeln ihr Wohlbefinden an. Durch solche Erfolge ermutigt, werden nun die großen Körbe aufgelöst und die Orchideen neu aufgebunden, denn in dem reichlichen, tiefgründigen Substrat hatte sich der Farn eingenistet, feste Rhizommassen gebildet und so üppiges Blattwerk entwickelt,

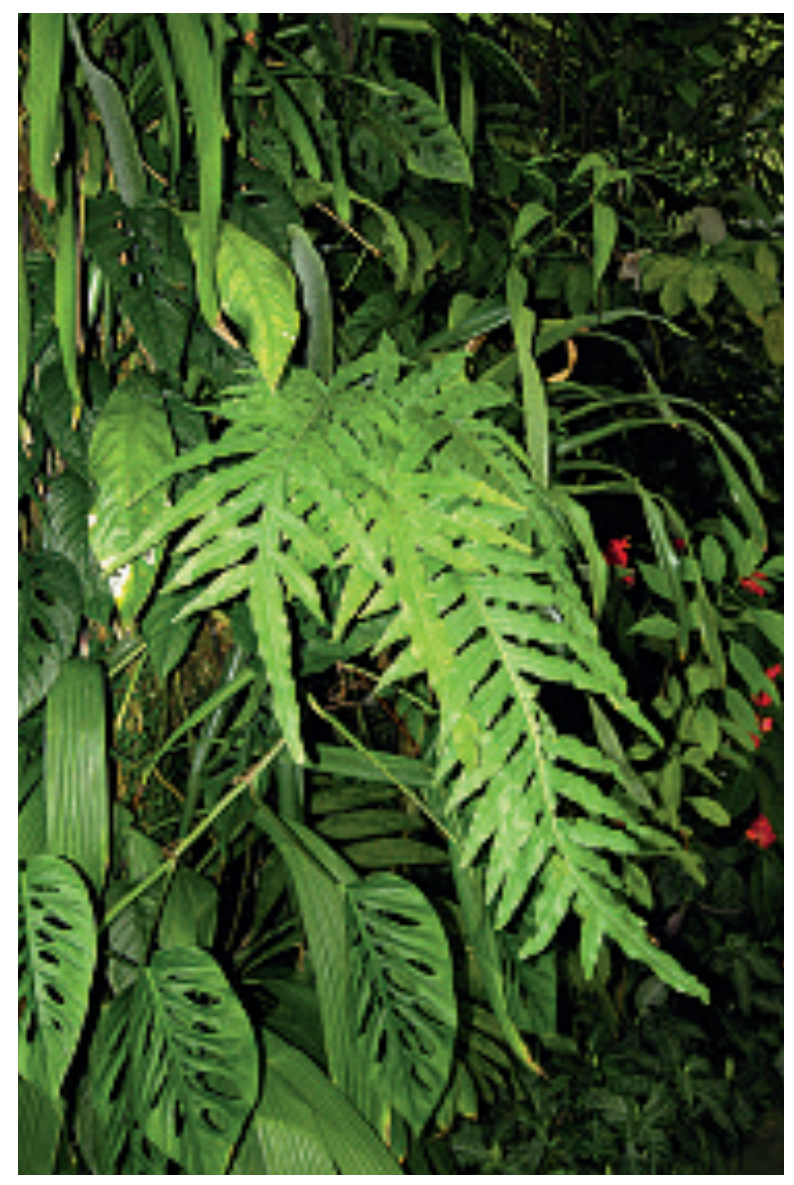

dass er selbst starke Angraecum und Vanda zu ersticken drohte. Ein hübscher tropisch-epiphytischer Farn zeigt, was in ihm steckt, erweist sich als Eroberer und Trockenkünstler und verwandelt sich in ein ausuferndes Problem - „das Leben findet einen Weg“.

\section{Literatur}

Bonstedt, C. (Hrsg.) 1931-1932: „Pareys Blumengärtnerei“. Beschreibung, Kultur und Verwendung der gesamten Gärtnerischen Schmuckpflanzen. 2 Bände. Berlin, Paul Parey.

Schneider, H., Schuettpelz, E., Pryer, K. M., Cranfill, R., Magallón, S. \& Lupia, R. ( 2004 ) Ferns diversified in the shadow of Angiosperms. - Nature 428: 553-557.

Schwerdtfeger, M. 2009: Anzucht von Farnen aus Sporen. - Palmengarten 73: 141-145. 